\section{Análisis de la hidatidosis humana en la Región de Coquimbo entre los años 2008 y 2012}

\author{
ANDREA SOTO-AGUILAR ${ }^{1, \mathrm{a}}$, TANIA JUNOD $^{1, \mathrm{a}, \mathrm{b}}$, MAURICIO CAMPILLAY $^{1, \mathrm{a}}$, \\ GERARDO ACOSTA-JAMETT ${ }^{2, a, b, c}$, CARLOS LANDAETA-AQUEVEQUE $^{1, a, c}$
}

\section{Analysis of human cyst echinococcosis in a northern Chilean region from 2008 to 2012}

Background: The Coquimbo Region in Chile has the highest incidence of hydatidosis in central and northern Chile. Aim: To analyze the incidence of human cystic echinococcosis (CE) in the Coquimbo Region between 2008 and 2012. Material and Methods: Official records of reported cases were analyzed. The association of the location, age and sex with the presence/absence of CE was analyzed by means of logistic regressions. The incidence was reported as cases per 100,000 inhabitants. Results: The mean annual incidence was 2.6 cases per 100,000 inhabitants, which ranged from 3.1 (2010) to 1.9 (2012) cases. The districts with higher incidence were Punitaqui (21.2 cases), Paihuano (9 cases), Rio Hurtado (8 cases), Canela (8.1 cases), Monte Patria (7.9 cases), Vicuña (6.9 cases) and Combarbalá (6.9 cases). The incidence in these locations was significantly higher than elsewhere in the region. Males had a significantly higher incidence than females (3.3 and 1.8 respectively). Incidence increased significantly with age, notably increasing after 45 years of age. Conclusions: Rural locations had the highest incidences of CE in the Coquimbo Region. The disease was reported more commonly in adults and mainly in men.

(Rev Med Chile 2017; 145: 603-609)

Key words: Chile; Echinococcosis; Incidence; Parasites; Rural Population.

\author{
${ }^{1}$ Facultad de Ciencias Veterinarias, \\ Universidad de Concepción, \\ Chillán, Chile. \\ ${ }^{2}$ Facultad de Ciencias Veterinarias, \\ Universidad Austral de Chile, \\ Valdivia, Chile. \\ ${ }^{a}$ Médico Veterinario. \\ ${ }^{\mathrm{b}} \mathrm{MsC}$ \\ CPhD. \\ El presente estudio fue \\ financiado por el Laboratorio de \\ Parasitología, Dr. Luis Rubilar, \\ de la Facultad de Ciencias \\ Veterinarias de la Universidad de \\ Concepción.
}

Recibido el 25 de enero de 2017, aceptado el 16 de mayo de 2017 .

Correspondencia a:

Carlos Landaeta-Aqueveque Vicente Méndez 595, Casilla 537, Chillán, Chile.

Teléfono: +56422207565

Fax: +56 422273201

clandaeta@udec.cl

\section{L}

a hidatidosis o equinococosis quística es una zoonosis parasitaria producida por cestodos del complejo Echinococcus granulosus (sensu lato), complejo de especies de cestodos cuyo hospedero definitivo es el perro y diversos mamíferos, incluido el humano, que pueden albergar su estado larval o hidatide ${ }^{1}$. Es una enfermedad cosmopolita, propia de sectores ganaderos ${ }^{2}$, y es considerada por la Organización Mundial de la Salud (OMS) como enfermedad desatendida ${ }^{3}$, afectando principalmente a personas que viven en condiciones socioeconómicas de pobreza, bajo nivel educativo y carencia de acceso a servicios sanitarios básicos ${ }^{4}$.

La hidatidosis tiene gran importancia en la salud pública, dada su alta tasa de incidencia en algunas regiones y elevado impacto en la calidad de vida de quienes la padecen, los costos ganaderos $y$ el costo en los sistemas de salud ${ }^{5,6}$.

En Chile, la hidatidosis está incluida dentro de las enfermedades de notificación obligatoria universal diaria, como se establece en el DS 158/047. El diagnóstico definitivo se logra con, al menos, una de tres alternativas: la evaluación de quistes extraídos por cirugía, dos pruebas serológicas distintas -una de alta sensibilidad (ELISA) y otra de alta especificidad (Western Blot)- $\mathrm{o}$ análisis moleculares ${ }^{8}$. La sospecha se establece mediante imagenología, o por estudios serológicos de alta sensibilidad ( $\sin$ incluir los de alta especificidad ${ }^{8}$.

La hidatidosis se presenta con mayor frecuen- 
cia en las regiones del sur del país y la Región de Coquimbo, en el norte; esta última es la que ha presentado la mayor tasa de incidencia entre las regiones de Arica y Parinacota y del Biobío ${ }^{5}$. En esta región, la prevalencia de equinococosis canina se ha estimado en $7,2 \%{ }^{9}$ y en animales de abasto varía entre $5,9 \%$ (caprinos) y $23,9 \%$ (bovinos) ${ }^{10}$.

La hidatidosis humana en la Región de Coquimbo es favorecida por la existencia de un gran número de animales de abasto mantenidos en las zonas rurales, la pobreza económica visible en muchas localidades y el menor acceso a los servicios de salud, dada la dificultad para viajar desde localidades distantes y aisladas ${ }^{11-13}$. Entre las provincias de esta región, la del Limarí es la que presentó la mayor tasa de incidencia entre los años 1995 y 2006, superando los 8 casos por cada 100.000 habitantes ${ }^{10}$; hasta la fecha no hay estudios de incidencia a nivel de comunas.

Dado que es una enfermedad crónica, se diagnostica más frecuentemente en personas adultas $^{12,14,15}$, observándose que, de los casos registrados en Chile, la mayor cantidad está entre los 30 y 59 años de edad ${ }^{16}$. Del mismo modo, con respecto al género, se ha observado en Chile que, de los casos reportados, la mayor cantidad corresponde a hombres ${ }^{15,16}$. Estos análisis basados en los casos registrados sugieren que la tasa de incidencia de esta enfermedad varía según género y edad. Por lo anterior, se definió como objetivo de este trabajo analizar la tasa de incidencia de la hidatidosis en la Región de Coquimbo entre los años 2008 y 2012, evaluando su variación según género, edad y las localidades.

\section{Material y Método}

Se utilizaron los registros del total de casos notificados de hidatidosis humana de la Región de Coquimbo, facilitados por la Unidad de Epidemiología de la Secretaría Regional Ministerial de Salud-Coquimbo. Estos registros no contenían la identidad de los pacientes y correspondieron a los casos reportados entre enero de 2008 y diciembre de 2012. Los casos negativos se obtuvieron por diferencia con el total de habitantes, según el censo $2002^{17}$. Se caracterizó la incidencia de la hidatidosis según las variables edad, género y localidad (comuna y provincia). Se evaluó la asociación entre presencia/ausencia de hidatidosis quística y edad, género y localidad (provincia) mediante regresión logística multifactorial, incluyendo estas últimas tres variables como variables independientes y la presencia/ausencia de hidatidosis como variable dependiente. En el caso de la variable localidad, se usó la provincia con mayor incidencia como categoría base y las otras dos fueron dicotomizadas (pertenecer o no pertenecer a la provincia) y tratadas como variables 'dummies'. En el caso de la edad, los habitantes se agruparon en rangos de 5 años (Figura 1) y se consideró como variable cuantitativa. El mejor modelo se decidió mediante prueba de razón de verosimilitud, de modo que, si al eliminar una variable la razón entre la verosimilitud del modelo más simple y el modelo más complejo es significativamente menor que 1 , no se elimina la variable al considerarse significativa la pérdida de verosimilitud. Finalmente, y dado lo observado en los resultados, se hizo una regresión logística dicotomizando la edad en dos grupos, mayores y menores de 45 años. Para realizar estos análisis se utilizó el software Stata $11 \mathrm{SE}^{\circledR}$ (Stata Corp. Ltda.). Las tasas de incidencia se informan como casos por cada $10^{5}$ habitantes (en adelante, sólo "casos").

\section{Resultados}

La Región de Coquimbo presentó una incidencia anual promedio de 2,56 casos, no observándose tendencia hacia el aumento o la disminución de las tasas de incidencia o mortalidad con el paso de los años (Tabla 1).

\section{Incidencias por provincias}

De las tres provincias de la Región de Coquimbo, la del Limarí fue la que presentó la mayor tasa de incidencia promedio para el período completo (5,9 casos), siendo significativamente menores las del Elqui y del Choapa (Tabla 2). Las incidencias anuales siguieron el mismo comportamiento casi todos los años (Tabla 1).

\section{Incidencias por comuna}

En la provincia del Elqui, la comuna de Higuera no presentó casos, mientras que, entre las demás comunas, en Coquimbo se reportó la menor incidencia. En contraste, la comuna de Paihuano presentó casos sólo los años 2009 y 2012, alcanzando las tasas anuales más elevadas de la provincia. 
Hidatidosis humana en la Región de Coquimbo, 2008-2012 - A. Soto-Aguilar et al

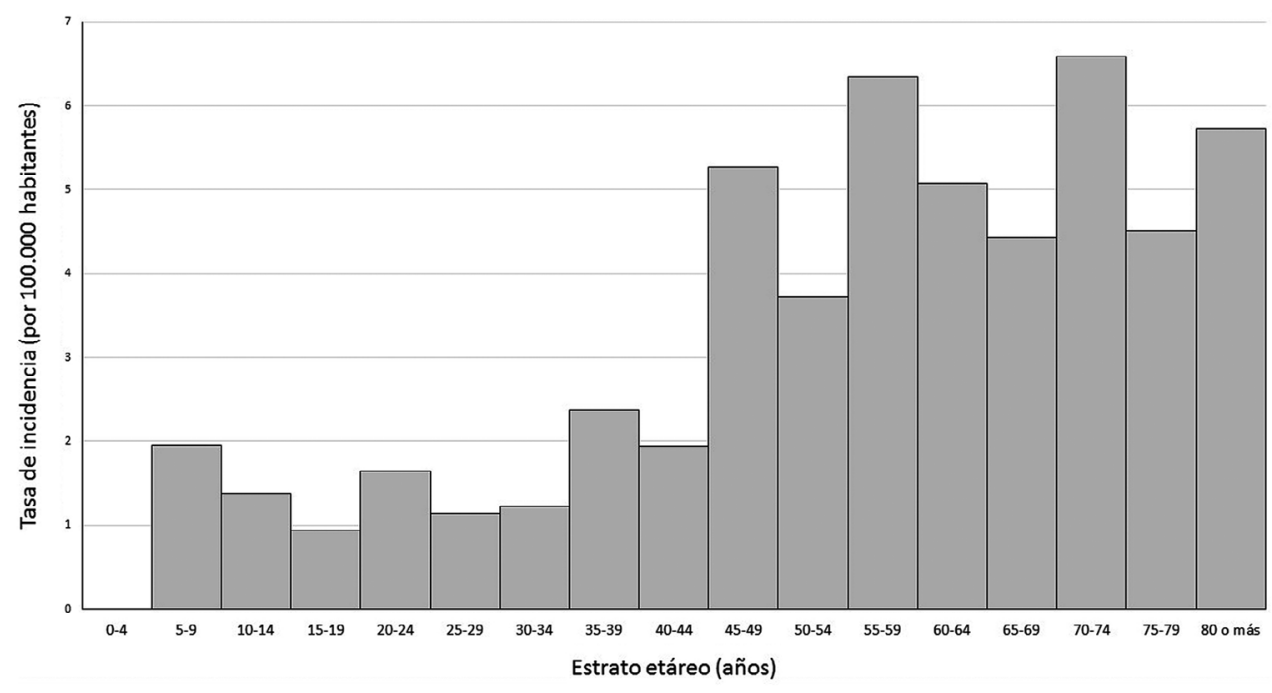

Figura 1. Tasa de incidencia (por cada 100.000 habitantes) de hidatidosis humana, por grupo etario, en la Región de Coquimbo entre los años 2008 y 2012.

Tabla 1. Casos notificados y tasas de incidencia (por cada 100 mil habitantes)* anuales de hidatidosis humana en las provincias y comunas de la Región de Coquimbo, y por género, entre los años 2008 y 2012

\begin{tabular}{|c|c|c|c|c|c|c|}
\hline Año & 2008 & 2009 & 2010 & 2011 & 2012 & $\begin{array}{l}\text { Promedio } \\
\text { del período }\end{array}$ \\
\hline \multicolumn{7}{|l|}{ Estadísticas regionales } \\
\hline Casos notificados & 17 & 18 & 21 & 17 & 13 & 17 \\
\hline Tasa incidencia & 2,5 & 2,7 & 3,1 & 2,6 & 1,9 & 2,6 \\
\hline \multicolumn{7}{|c|}{ Tasas de incidencias locales } \\
\hline Provincia del Elqui & 0,5 & 2,3 & 0,9 & 2 & 0,9 & 1,3 \\
\hline La Serena & 0,5 & 2,6 & 1,5 & 2 & 0 & 1,3 \\
\hline Higuera & 0 & 0 & 0 & 0 & 0 & 0 \\
\hline Paihuano & 0 & 22,7 & 0 & 0 & 22,3 & 9 \\
\hline Vicuña & 0 & 15,5 & 0 & 7,6 & 11,4 & 6,9 \\
\hline Andacollo & 0 & 0 & 9 & 0 & 0 & 1,8 \\
\hline Coquimbo & 0,5 & 0 & 0 & 1,4 & 0 & 0,4 \\
\hline Provincia del Limarí & 8,2 & 4,6 & 9,6 & 2,8 & 4,4 & 5,9 \\
\hline Ovalle & 4,6 & 4,5 & 3,5 & 3,5 & 2,6 & 3,7 \\
\hline Monte Patria & 12,3 & 0 & 21,3 & 3 & 3 & 7,9 \\
\hline Río Hurtado & 20,1 & 20,1 & 0 & 0 & 0 & 8 \\
\hline Punitaqui & 20,3 & 19,1 & 38,3 & 0 & 28,3 & 21,2 \\
\hline Combarbalá & 14,2 & 0 & 13,5 & 0 & 6,7 & 6,9 \\
\hline Provincia del Choapa & 1,1 & 1,1 & 1,1 & 5,6 & 2,2 & 2,2 \\
\hline Illapel & 3,1 & 0 & 0 & 3,1 & 0 & 1,2 \\
\hline Canela & 0 & 0 & 0 & 20,3 & 20,2 & 8,1 \\
\hline Los Vilos & 0 & 0 & 0 & 0 & 0 & 0 \\
\hline Salamanca & 0 & 3,8 & 3,8 & 7,5 & 0 & 3 \\
\hline \multicolumn{7}{|c|}{ Tasas de incidencia por género } \\
\hline Masculino & 2,7 & 4,9 & 2,6 & 4,1 & 2,2 & 3,3 \\
\hline Femenino & 2,3 & 0,6 & 3,6 & 1,1 & 1,6 & 1,8 \\
\hline
\end{tabular}

*Tasas calculadas sobre la base de la población para la Región de Coquimbo, según censo 2002. 
Tabla 2. Resultados de la regresión logística multifactorial que evalúa la asociación entre tres variables (Provincia, género y edad) y la presencia de hidatidosis en habitantes de la Región de Coquimbo entre los años 2008 y 2012

\begin{tabular}{|lccc|}
\hline & OR & p & Intervalo de confianza \\
\hline Provincia (Elqui*) & 0,23 & $<0,001$ & $0,14-0,36$ \\
\hline Provincia (Choapa*) & 0,37 & 0,004 & $0,19-0,73$ \\
Género (masculino*) & 1,82 & 0,006 & $1,18-2,78$ \\
Edad & 1,14 & $<0,001$ & $1,09-1,20$ \\
\hline
\end{tabular}

*Variables categóricas: la categoría base para Provincia es Limarí y para género es femenino. Las categorías mencionadas entre paréntesis corresponden a las categorías de comparación; OR > a 1 indica mayor disparidad ('odds') para esas categorías.

De las cinco comunas de la provincia del Limarí, la comuna de Punitaqui fue la que presentó las mayores tasas anuales de la provincia y de la región. La comuna de Río Hurtado sólo presentó casos el año 2008 y 2009, pero con altas tasas en ambos años. En la provincia del Choapa, la comuna de Los Vilos no presentó casos en los cinco años del estudio. En contraste, la comuna de Canela sólo presentó casos los años 2011 y 2012, con las tasas anuales más elevadas de la provincia (Tabla 1 ).

\section{Asociaciones de variables}

Las tres variables, género, edad y localidad (provincia), mostraron asociación significativa con la presencia de hidatidosis, siendo más frecuente la presentación en hombres, en la medida que aumenta la edad y en los habitantes de la provincia del Limarí, respecto de las otras provincias de la región. La Tabla 2 muestra los resultados de la regresión logística. Al eliminar la variable con menor significación, la verosimilitud del modelo se redujo significativamente $\left(\chi^{2}(1\right.$ g. 1.$)=7,79$, $\mathrm{p}=0,0053)$, considerándose todas las variables como factores significativos.

Considerando todos los años, el género masculino presentó una incidencia significativamente mayor que el género femenino (Tablas 1 y 2). Sin embargo, esta tendencia no fue constante a lo largo de los 5 años incluidos en el estudio (Tabla 1), pero sí se mantuvo en todas las provincias (Tabla 3 ).

La edad se asoció positivamente con la presentación de hidatidosis (Tabla 2), siendo el grupo de 70 a 74 años el que presentó la más elevada tasa de incidencia, observándose también un alza importante en la incidencia a partir de los 45 años de edad (Figura 1). Al dicotomizar la variable edad
Tabla 3. Tasa de incidencia (por cada $100 \mathrm{mil}$ habitantes) de hidatidosis humana en la Región de Coquimbo entre los años 2008 y 2012. Distribución por provincia y género*

\begin{tabular}{|lcc|}
\hline Provincia & \multicolumn{2}{c|}{ Género } \\
\hline Elqui & Masculino & Femenino \\
\hline Limarí & 1,9 & 0,7 \\
Choapa & 6,8 & 4,9 \\
\hline
\end{tabular}

*Tasa de incidencia calculada sobre la base de la población para la Región de Coquimbo, según censo 2002.

entre mayores y menores de 45 años, se observó que en mayores de 45 años la presentación fue significativamente más frecuente que en menores de 45 años (OR: 3,68; p < 0,001; I. C.: 2,40-5,62).

\section{Discusión}

Si bien, la tasa de incidencia anual promedio, 2,6 casos, es menor a la reportada para el período 1995 a 2006 de 4,1 casos $^{10}$, en todos los años se observó una incidencia superior a la incidencia promedio del país para 2012 (1,6 casos), último año considerado en este estudio.

De las 15 comunas de la región, la comuna con mayor tasa de incidencia promedio para los años de estudio fue Punitaqui, cuya tasa promedio superó en más de dos veces las de las otras comunas (Tabla 3). Estudios previos en esta comuna han reportado también altos niveles de prevalencia de hidatidosis humana $(6,8 \%)^{11}$ y echinococcosis 
canina $(20 \%)^{18}$. Las otras comunas con alta incidencia fueron Río Hurtado y Monte Patria en la provincia de Limarí, Paihuano en la provincia del Elqui, y Canela en la provincia de Choapa. Estas son precisamente las comunas con los mayores índices de ruralidad en sus respectivas provincias ${ }^{19}$, característica que se ha identificado como factor de riesgo para la hidatidosis ${ }^{20}$.

De las tres provincias de la región, la del Limarí fue la que presentó las mayores tasas de incidencia anuales, manteniendo la tendencia reportada entre 1995 y $2006^{10}$, aunque con tasas un poco menores $(5,9$ versus 8,5 casos reportados en el estudio previo). Esta es la provincia que ha presentado también la mayor tasa de seroprevalencia de hidatidosis humana, considerando las localidades rurales (3,6\% versus 2,7\% en la provincia del Elqui y $1 \%$ en la provincia del Choapa ${ }^{14}$. Esto puede ser favorecido por la alta proporción de personas viviendo en condiciones de pobreza en que su principal actividad económica es la ganadería familiar de autoconsumo, sumado a otros factores de riesgo, como las malas prácticas en la tenencia de los perros, principalmente la no desparasitación y la alimentación de éstos con vísceras crudas, provenientes de la faena doméstica de animales ${ }^{11}$. Debido a esto, se ha hecho necesario considerar medidas en forma prioritaria dado el impacto de esta enfermedad para la salud pública en la provincia del Limarí. Es así como, desde el año 2010, se está desarrollando un Programa de Prevención y Control de Hidatidosis, el que incluye el registro, la desparasitación y esterilización de perros, y la educación sanitaria ${ }^{21}$.

La mayor incidencia en hombres que en mujeres es consistente con estudios previos que también reportan mayores casos de hidatidosis en el género masculino ${ }^{15,22-24}$; sin embargo, también hay estudios que han reportado lo contrario en otros países ${ }^{25}$, sugiriendo que hay características culturales locales que pueden determinar el efecto del género en la presencia de la enfermedad. Es necesario, entonces, que en futuras investigaciones se indague en las causas que expliquen la diferencia de la incidencia entre hombres y mujeres; las cuales pueden yacer, por ejemplo, en las actividades más frecuentemente realizadas por cada género o en actitudes, conductas o prácticas distintas según género; con el fin de poder diseñar estrategias de prevención y educación que consideren dichas causas.
La incidencia fue mayor conforme aumenta la edad, lo que es consistente con lo encontrado tanto en otras localidades de Chile ${ }^{26,27}$ como también en otros países ${ }^{25}$. Esto puede estar dado por el carácter de cronicidad de la enfermedad; no obstante, es llamativo el cambio en la tasa de incidencia a partir de los 45 años. Es difícil sostener alguna hipótesis acerca de la causa de este resultado, puesto que el período de incubación suele ser variable, entre meses y años ${ }^{28}$, dificultando el planteamiento de que a partir de una determinada edad puedan ocurrir con mayor frecuencia las infecciones. Dado que los resultados se basan en notificaciones oficiales de casos confirmados, cabe la pregunta si a partir de los 45 años los casos probables son tratados con mayor urgencia, provocándose de esta manera esta alza en la frecuencia de casos confirmados. Nuevos estudios que analicen más detalladamente la asociación entre edad e infección permitirán buscar con más precisión las causas que expliquen el aumento de casos confirmados a mayor edad, de modo de enfocar las medidas de prevención y educación de acuerdo con esas causas.

Si bien, la no desparasitación de perros es un factor de riesgo en la región ${ }^{11}$, en la literatura se menciona que la administración de praziquantel en perros, a nivel de Sudamérica, no ha sido una medida suficiente ${ }^{29}$. Es necesario entonces enfocarse también en los factores de riesgo factibles de modificar, dentro de los cuales están algunas prácticas como alimentar a los perros con vísceras de animales ${ }^{11,30,31}$ o la correcta disposición de las heces de los perros ${ }^{11}$. En lo relacionado con el conocimiento de la enfermedad, se ha visto que el hecho que la población conozca las vías de transmisión desde el perro y el modo de infección de éste, es un factor protector ${ }^{31}$. Todo lo anterior sugiere la importancia de la educación en la prevención de la hidatidosis, la que debe ser incorporada de manera periódica y a largo plazo para modificar las conductas en la población, especialmente en las comunas con mayores tasas de incidencia.

\section{Referencias}

1. Nakao M, Lavikainen A, Yanagida T, Ito A. Phylogenetic systematics of the genus Echinococcus (Cestoda: Taeniidae). Int J Parasitol 2013; 43: 1017-29.

2. Apt W, Pérez C, Galdámez E, Campano S, Vega F, Vargas D, et al. Equinococosis/hidatidosis en la VII Región 
de Chile: diagnóstico e intervención educativa. Rev Panam Salud Publica 2000; 7: 8-16.

3. OMS. Notas descriptivas: enfermedades tropicales desatendidas. 2015. Disponible en: http://www.who. int/topics/tropical_diseases/factsheets/neglected/es/ [Consultado el 20 de mayo de 2016].

4. OMS. Enfermedades tropicales desatendidas: preguntas más frecuentes. 2010. Disponible en: http://www.who. int/topics/tropical_diseases/qa/faq/es/ [Consultado el 20 de mayo de 2016].

5. Cortés S, Valle C. Hidatidosis humana: Generalidades y situación epidemiológica en Chile según egresos hospitalarios y notificación obligatoria entre los años 2001 y 2005. Rev Chilena Infectol 2010; 27: 329-35.

6. Farías CMJ, Medina GA. Estimación del costo para el diagnóstico precoz de la hidatidosis en niños de $5 \mathrm{y}$ 12 años en la provincia de Nuble, Región del Bío-Bío, Chile. Rev Chilena Infectol 2011; 28: 50-6.

7. ISP. Reglamento sobre notificación de enfermedades transmisibles de declaración obligatoria. 2004. Disponible en: http://www.ispch.cl/ley20285/t_activa/marco_normativo/7c/dec_158_04.pdf [Consultado el 27 de mayo de 2016].

8. MINSAL. Hidatidosis (equinococosis quística) Definiciones de caso. 2016. Disponible en: http://epi.minsal. cl/hidatidosis-definiciones-de-caso/ [Consultado el 12 de abril de 2016].

9. Acosta-Jamett G, Cleaveland S, Bronsvoort BMd, Cunningham AA, Bradshaw H, Craig PS. Echinococcus granulosus infection in domestic dogs in urban and rural areas of the Coquimbo region, north-central Chile. Vet Parasitol 2010; 169: 117-22.

10. Acosta-Jamett G, Cleaveland S, Cunningham AA, Bronsvoort BMd, Craig PS. Echinococcus granulosus infection in humans and livestock in the Coquimbo region, north-central Chile. Vet Parasitol 2010; 169: 102-10.

11. Acosta-Jamett G, Weitzel T, Boufana B, Adones C, Bahamonde $\mathrm{A}, \mathrm{Abarca} \mathrm{K}$, et al. Prevalence and risk factors for echinococcal infection in a rural area of Northern Chile: a household-based cross-sectional study. PLoS Negl Trop Dis 2014; 8: e3090.

12. Martínez P. Caracterización de la mortalidad por hidatidosis humana: Chile, 2000-2010. Rev Chilena Infectol 2014; 31: 7-15.

13. Wagstaff A. Pobreza y desigualdades en el sector de la salud. Rev Panam Salud Publica 2002; 11: 316-26.

14. Lorca M, Garrido M, Contreras MDC, Salinas P, Álvarez C, Ruiz M, et al. Seroprevalencia de hidatidosis humana en la Región de Coquimbo. Chile. Parasitol Latinoam 2006; 61: 111-6.
15. Acosta-Jamett G, Vargas R, Ernst S. Caracterización epidemiológica de equinococosis quística humana y animal en la Región de Los Ríos entre el período 19992009. Rev Chilena Infectol 2016; En prensa:

16. ISP. Vigilancia de hidatidosis. Chile, 2010-2014. 2015. Disponible en: http://www.ispch.cl/sites/default/files/ Bolet\%C3\%ADn\%20Hidatidosis\%2030-03-2015.pdf [Consultado el 12 abril de 2016].

17. INE. Censo de población y de vivienda 2002. 2005. Disponible en: http://www.ine.cl/canales/chile_estadistico/ censos/censo_poblacion_vivienda.php [Consultado el 2 de enero de 2016].

18. Coquimbo GORE. Cuenta pública regional 2010: Región de Coquimbo. 2011. Disponible en: http://www.gorecoquimbo.gob.cl/doc_transparencia/cuentas_publicas/ cp2010.pdf [Consultado el 12 abril de 2016].

19. Alvarado G, Moya JC. División político administrativa y censal 2007. 2008. Disponible en: http://www.ine.cl/ canales/chile_estadistico/territorio/division_politico_ administrativa/pdf/DPA_COMPLETA.pdf [Consultado el 12 de abril de 2016].

20. Possenti A, Manzano-Román R, Sánchez-Ovejero C, Boufana B, La Torre G, Siles-Lucas M, et al. Potential Risk Factors Associated with Human Cystic Echinococcosis: Systematic Review and Meta-analysis. Plos Negl Trop Dis 2016; 10 (11): e0005114.

21. SEREMI-Coquimbo. Programa de Prevención y Control de Hidatidosis en las comunas de Punitaqui, Monte Patria y Combarbalá, Provincia del Limarí, Región de Coquimbo. 2015. Disponible en: http://seremi4.redsalud.gob.cl/?p=5004 [Consultado el 27 de mayo de 2016].

22. Ernst S, Rodríguez E, Murua A, Ramírez G. [Analysis of some variables in patients with surgically treated hydatidosis in the Regional Hospital of Valdivia, Chile, 1976-1986]. Bol Chil Parasitol 1989; 44: 30-3.

23. Jans BJ, Bóquez MP, Marambio GA, Manoli SP, Hollstein GA, Zarges TP. Resultados del tratamiento de la hidatidosis pulmonar complicada y no complicada. Rev Chilena Cirugía 2012; 64: 346-51.

24. Tercero Gutiérrez MJ, Olalla Herbosa R. Hidatidosis. Una zoonosis de distribución mundial. Offarm 2008; 27: 88-94.

25. Wang Q, Huang Y, Huang L, Yu W, He W, Zhong B, et al. Review of risk factors for human echinococcosis prevalence on the Qinghai-Tibet Plateau, China: a prospective for control options. Infect Dis Poverty 2014; 3 : 1-8.

26. Martínez P. Hidatidosis humana: antecedentes generales y situación epidemiológica en Chile, 2001-2009. Rev Chil Infectol 2011; 28: 585-91.

27. Schenone H, Contreras MdC, Salinas P. Sandoval L, 
Saavedra T, Rojas A. Hidatidosis humana en Chile. Seroprevalencia y estimación del número de personas infectadas. Bol Chil Parasitol 1999; 54: 70-3.

28. OMS. Echinococcosis. 2016. Disponible en: http://www. who.int/mediacentre/factsheets/fs377/en/ [Consultado el 27 de mayo de 2016].

29. Larrieu E, Zanini F. Critical analysis of cystic echinococcosis control programs and praziquantel use in South America, 1974-2010. Rev Panam Salud Publica 2012; 31: 81-7.
30. Oba P, Ejobi F, Omadang L, Chamai M, Okwi A, Othieno E, et al. Prevalence and risk factors of Echinococcus granulosus infection in dogs in Moroto and Bukedea districts in Uganda. Trop Anim Health Prod 2015; 1-6.

31. Li D, Gao Q, Liu J, Feng Y, Ning W, Dong Y, et al. Knowledge, attitude, and practices (KAP) and risk factors analysis related to cystic echinococcosis among residents in Tibetan communities, Xiahe County, Gansu Province, China. Acta Trop 2015; 147: 17-22.

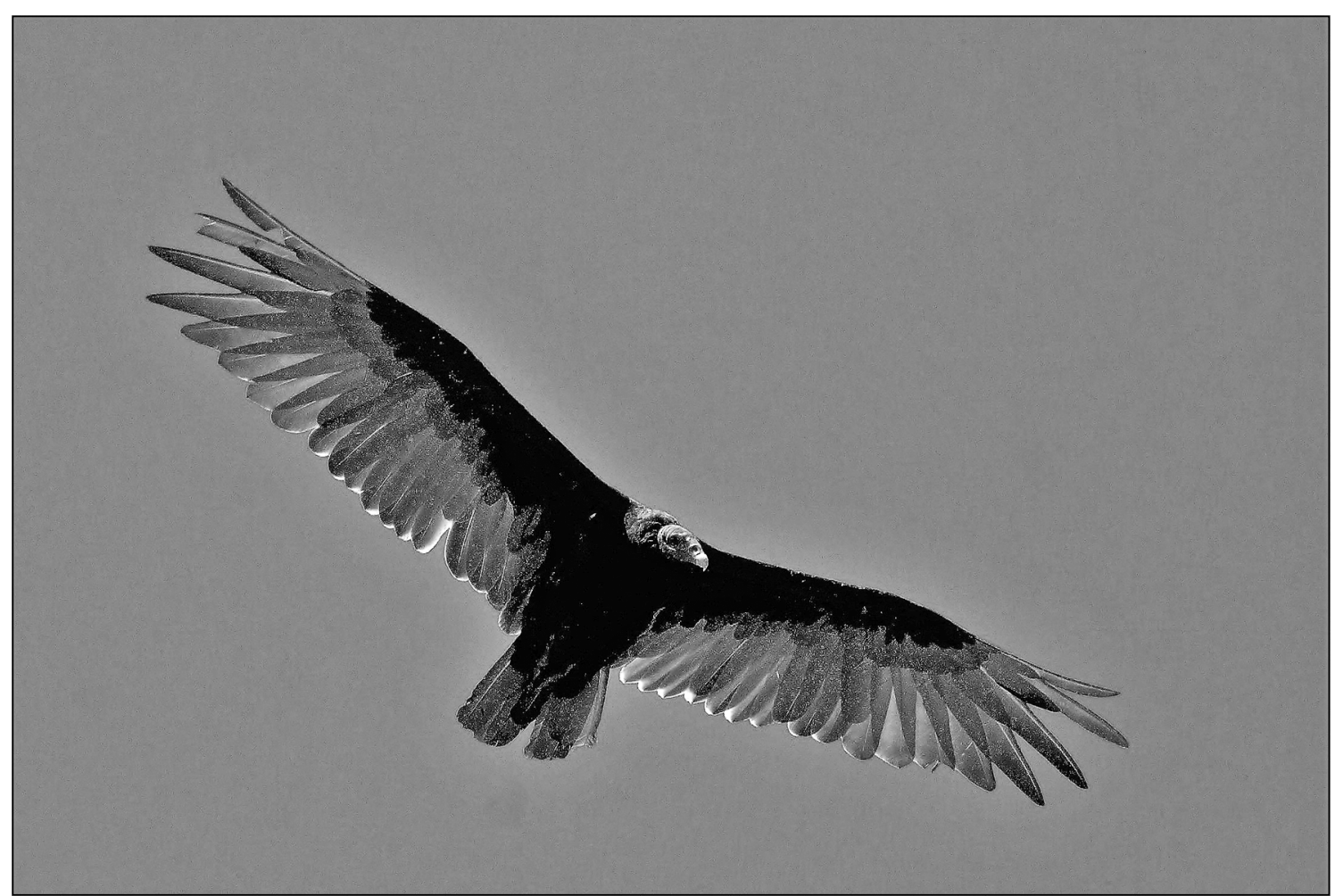

Cathartes Aura (Gallinazo). Dr. Jorge Sapunar Zenteno 